\title{
Lift zonoids, random convex hulls and the variability of random vectors
}

\author{
GLEB KOSHEVOY $^{1}$ and KARL MOSLER ${ }^{2 *}$ \\ ${ }^{I}$ Central Institute of Mathematics and Economics, Russian Academy of Science, Krasikova 32, \\ Moscow 117418, Russia \\ ${ }^{2}$ Seminar für Wirtschafts- und Sozialstatistik, Universität zu Köln, D-50923 Köln, Germany. \\ E-mail:mosler@wiso.uni-koeln.de
}

For a $d$-variate measure a convex, compact set in $\mathbb{R}^{d+1}$, its lift zonoid, is constructed. This yields an embedding of the class of $d$-variate measures having finite absolute first moments into the space of convex, compact sets in $\mathbb{R}^{d+1}$. The embedding is continuous, positive homogeneous and additive and has useful applications to the analysis and comparison of random vectors. The left zonoid is related to random convex sets and to the convex hull of a multivariate random sample. For an arbitrary sampling distribution, bounds are derived on the expected volume of the random convex hull. The set inclusion of lift zonoids defines an ordering of random vectors that reflects their variability. The ordering is investigated in detail and, as an application, inequalities for random determinants are given.

Keywords: convex sets; dilation; measure metric; ordering of distributions; random determinants; random convex sets

\section{Introduction}

To analyse and compare random vectors, we propose a new geometric approach. For any $d$ variate measure, a convex set in $\mathbb{R}^{d+1}$, its lift zonoid, is constructed. This yields an embedding of the space of measures on $\mathbb{R}^{d}$ that have finite absolute first moments into the space of convex, compact sets in $\mathbb{R}^{d+1}$. The embedding is continuous, positive homogeneous and additive; continuous with respect to weak bounded convergence of measures and the Hausdorff distance of sets, and additive with respect to the usual sum of measures and the Minkowski sum of sets. In this way, the distribution of a random vector is represented by its lift zonoid.

The lift zonoid representation proves to be very useful. Here we develop two streams of applications. First, the lift zonoid is related to random convex sets and to the convex hull of a multivariate random sample. Second, the set inclusion of lift zonoids defines an ordering of random vectors that reflects their variability.

Recent interest in zonoids has arisen from some surprising connections between convex geometry, analysis (positive definite forms, Radon transforms, vector measures), and stochastic geometry (stochastic processes). A recent survey is Goodey and Weil (1993). The

\footnotetext{
* To whom correspondence should be addressed.
} 
zonoid of a multivariate probability measure (or random vector) has been investigated by Rickert (1967), Schneider (1967) and others.

A random convex set is a random variable in the space of non-empty, convex, compact subsets of $\mathbb{R}^{d}$. It has a set-valued expectation (e.g., Weil and Wieacker 1993). In particular, if $X$ is a $d$-variate random vector, then the random segment $[\mathbf{0}, X]$ that extends from the origin to the random point $X$ is a random convex set in $\mathbb{R}^{d}$. We show that its expectation equals the zonoid of the distribution of $X$. The lift zonoid of $X$ comes out to be the expectation of the random segment

$$
\left[\mathbf{0},\left(\begin{array}{c}
1 \\
X
\end{array}\right)\right]
$$

in $\mathbb{R}^{d+1}$. It contains the origin and is symmetric around the point

$$
\frac{1}{2}\left(\begin{array}{c}
1 \\
\mu
\end{array}\right)
$$

where $\mu$ denotes the expectation vector of $X$. In contrast to the zonoid, the lift zonoid of a measure does determine the underlying measure in a unique way and, therefore, yields a useful embedding.

The convex hull of independent and identically distributed random points has been studied by many authors, beginning with Rényi and Sulanke $(1963 ; 1964)$ and Efron $(1965)$. By means of the lift zonoid approach we derive upper and lower bounds on the expected volume of the random convex hull. The bounds are based on the volume of the lift zonoid of the sampling distribution. They apply to any distribution that has finite expectation.

The problem of comparing random vectors with respect to their variability is common to many parts of applied probability and statistics, among them estimation problems and the comparison of experiments (Torgersen 1991). We introduce the lift zonoid ordering among probability distributions (or random vectors), characterize it in many respects and show that it is a variability ordering weaker than dilation. The lift zonoid ordering is easy to handle and allows the derivation of interesting probability inequalities.

In Section 2 we investigate the zonoid of a finite measure in $\mathbb{R}^{d}$. Section 3 presents the definition and theory of the lift zonoid. Consider the space of convex compacts in $\mathbb{R}^{d+1}$, endowed with the Minkowski sum and the Hausdorff distance. We demonstrate that any measure that has finite absolute first moments is, by its lift zonoid, mapped into this space and that the mapping is injective, positive linear, and continuous, hence a homoeomorphic embedding. Given two measures, the inclusion of their lift zonoids is characterized by conditions on the univariate projections of these measures. A new metric among measures is proposed. In Section 4 we derive bounds on the expected volume of a random convex hull, i.e., the convex hull of a $d$-variate random sample, for a general sampling distribution. To this end, we introduce the notion of a random lift zonotope and show that, up to a known constant, its expected volume equals the volume of the lift zonoid. The result is used to provide upper and lower bounds for the expected volume of the random convex hull in terms of the volume of the lift zonoid. Section 5 contains a comprehensive investigation of the ordering among random vectors that is induced by the inclusion of lift zonoids. The ordering between two random vectors is characterized by expectation inequalities and shown 
to be equivalent to the dilation ordering of all univariate projections of the two vectors. Thus the lift zonoid order is an ordering of variability, and weaker than the multivariate dilation order. The lift zonoid order is continuous. It is preserved under probability mixtures and convex convolutions and under arbitrary affine transformations. In particular, the lift zonoid order between two random vectors implies the same order between all their marginals. A reverse result is true under stochastic independence. Further, we show that every probability distribution, in the weak sense, can be approximated by empirical distributions that are below it and by absolutely continuous distributions that are above it (in the lift zonoid order). As a final application, inequalities for the expectation of random determinants are derived.

We conclude this section with some notation. A point $x$ in Euclidean space $\mathbb{R}^{k}$ is a column, $x^{\mathrm{T}}$ denotes the transpose of $x$, and $\langle x, y\rangle$ the usual inner product. $\mathbb{R}_{+}^{k}$ is the subset of points in $\mathbb{R}^{k}$ that have non-negative components, and $S^{k-1}$ the unit sphere. $\mathscr{S}^{k}$ denotes the Borel sets in $\mathbb{R}^{k}, \mathbf{0}$ is the origin, $[0, x]=\{y: y=\alpha \cdot x, \alpha \in[0,1]\}$ the segment from the origin to $x, K(\epsilon)$ the $\epsilon$-ball around the origin. For two sets $C$ and $D, C \oplus D=$ $\{x+y: x \in C, y \in D\}$ is their Minkowski sum, and $\delta(C, D)=\inf \{\epsilon: C \subset D \oplus K(\epsilon)$, $D \subset C \oplus K(\epsilon), \epsilon>0\}$ is their Hausdorff distance. For random variables, $=_{\mathrm{d}}$ means equality in distribution.

\section{The zonoid of a measure}

Let $\mathscr{C}$ be the class of non-negative measures $F$ on $\left(\mathbb{R}^{d}, \mathscr{B}^{d}\right)$ that have finite positive total mass $\alpha(F)=\int \mathbb{R}^{d} \mathrm{~d} F(x)>0$, and let $\mathscr{F} \subset \mathscr{l}$ be the class of probability measures. $F$ is called absolutely continuous (discrete) if it possesses a density with respect to Lebesgue (counting) measure on $\mathbb{R}^{d}$. Let $\mathscr{C}_{0}\left(\mathscr{F}_{0}\right)$ be the subclass of measures (probability measures) $F$ for which $\int_{\mathbb{R}^{d}}\|x\| \mathrm{d} F(x)$ is finite. The zonoid of a measure is defined as follows.

Definition 2.1. Let $F \in \mathscr{l}$ be given. For a measurable function $g: \mathbb{R}^{d} \rightarrow[0,1]$, consider the point $\zeta(F, g)=\left(z_{1}, \ldots, z_{d}\right)^{\mathrm{T}} \in \mathbb{R}^{d}$,

$$
\zeta(F, g)=\left(\int_{\mathbb{R}^{d}} g(x) x \mathrm{~d} F(x)\right) .
$$

The set

$$
Z(F) \equiv\left\{\zeta(F, g): g: \mathbb{R}^{d} \rightarrow[0,1] \text { measurable }\right\}
$$

is called the zonoid of $F$.

Let us recall several properties of zonoids. A compact zonoid is a limit, in the Hausdorff sense of sums of line segments. The sum of two zonoids is a zonoid. The linear image of a zonoid is a zonoid. In particular, a projection of a zonoid is a zonoid.

Zonoids of measures have the following properties. 
Proposition 2.1. Let $F \in \mathscr{M l}$.

(i) The zonoid of $F$ is convex and contains $\mathbf{0}$.

(ii) If $F \in \mathscr{C l}_{0}$, the zonoid is compact and symmetric around $\frac{1}{2} \mu(F)$.

Further, $Z(F)$ is the convex hull of the range of the vector measure $\tau_{F}$, given by $\tau_{F}(A)=$ $\int_{A} x \mathrm{~d} F(x), A \in \mathscr{B}^{d}$. If $F$ is absolutely continuous then $Z(F)$ equals the range of $\tau_{F}$, according to Lyapunov's theorem (Lindenstrauss 1966). For the derivation of these properties, the reader is referred to Bolker (1969); see also Schneider and Weil (1983).

Given a convex, compact set $K \subset \mathbb{R}^{d}$, the support function $h(K, \cdot): \mathbb{R}^{d} \rightarrow \mathbb{R}$ of $K$ is defined by

$$
h(K, p)=\max \{\langle x, p\rangle: x \in K\}, \quad p \in \mathbb{R}^{d} .
$$

The function $h(K, \cdot)$ is continuous, convex and homogeneous of degree $1 . h(K, \cdot) \geqslant 0$ if and only if $\mathbf{0} \in K$. For two convex, compact sets $K$ and $R$ we have $h(K \oplus R, \cdot)=h(K, \cdot)+$ $h(R, \cdot), h(K, \cdot) \leqslant h(R, \cdot)$, if and only if $K \subset R$, and, in particular, $h(K, \cdot)=h(R, \cdot)$ if and only if $K=R$. For these and other properties of support functions, see Eggleston (1958). The following theorem shows what the support function of the zonoid of a measure looks like.

Theorem 2.1. For $F \in \mathscr{C l}_{0}$, the support function of $Z(F)$ is given by

$$
h(Z(F), p)=\int_{\{x:\langle x, p\rangle \geqslant 0\}}\langle x, p\rangle \mathrm{d} F(x)=\int_{\mathbb{R}^{d}} \max \{0,\langle x, p\rangle\} \mathrm{d} F(x) .
$$

Proof. For every measurable function $g: \mathbb{R}^{d} \rightarrow[0,1]$ and every $p \in \mathbb{R}^{d}$ we obtain

$$
g(x)\langle x, p\rangle \leqslant h_{p}(x)\langle x, p\rangle,
$$

where $h_{p}$ denotes the indicator function of the set $\{x:\langle x, p\rangle \geqslant 0\}$. Therefore

$$
\begin{aligned}
\langle\zeta(F, g), p\rangle & =\int_{\mathbb{R}^{d}} g(x)\langle x, p\rangle \mathrm{d} F(x) \leqslant \int_{\mathbb{R}^{d}} h_{p}(x)\langle x, p\rangle \mathrm{d} F(x) \\
& =\left\langle\zeta\left(F, h_{p}\right), p\right\rangle=\int_{\{x:\langle x, p\rangle \geqslant 0\}}\langle x, p\rangle \mathrm{d} F(x) \\
& =\int_{\mathbb{R}^{d}} \max \{0,\langle x, p\rangle\} \mathrm{d} F(x) .
\end{aligned}
$$

Because $\zeta\left(F, h_{p}\right) \in Z(F),(2.1)$ follows.

With this theorem we demonstrate that the zonoid of a probability measure is the expectation of a random segment. Recall the definition of a random convex set and its 
expectation. A random convex set $C$ is a Borel measurable map from a probability space $(\Omega, \mathscr{B}, P)$ to the space of non-empty, compact, convex subsets of $\mathbb{R}^{d}$. The expectation of a random convex set $C$ is the set $\mathrm{E}(C)$ given implicitly by

$$
h(\mathrm{E}(C), p)=\mathrm{E}(h(C, p)), \quad p \in \mathbb{R}^{d} .
$$

This set-valued expectation has appeared in different settings; see, for example, Weil and Wieacker (1993). If the norm of $C$ has finite expected value then $\mathrm{E}(C)$ is a compact set.

Proposition 2.2. Let $F \in \mathscr{F}_{0}$ and $X$ be a random vector distributed as $F$. Then $Z(F)=$ $\mathrm{E}([\mathbf{0}, X])$.

Proof. Because $Z(F)$ and $\mathrm{E}([\mathbf{0}, X)]$ are compact sets, they are equal if and only if they have the same support functions. According to (2.1), we have $h(Z(F), p)=\int_{\mathbb{R}^{d}} \max \{0$, $\langle x, p\rangle\} \mathrm{d} F(x)$. On the other hand, $h([\mathbf{0}, X], p)=\max \{0,\langle X, p\rangle\}$, hence

$$
h(\mathrm{E}([\mathbf{0}, X]), p)=\mathrm{E}(h([\mathbf{0}, X], p))=\int_{\mathbb{R}^{d}} \max \{0,\langle x, p\rangle\} \mathrm{d} F(x)=h(Z(F), p),
$$

and we are done.

Another consequence of Theorem 2.1 is the following:

Corollary 2.1. Let $F, G \in \mathscr{C}_{0}$. Then $Z(F)=Z(G)$ if and only if

$$
\int_{\{x:\langle x, p\rangle \geqslant 0\}} x \mathrm{~d} F(x)=\int_{\{x:\langle x, p\rangle \geqslant 0\}} x \mathrm{~d} G(x)
$$

for every $p \in \mathbb{R}^{d}$.

From Corollary 2.1 it follows that the zonoids of different measures may be the same. For example, if $d=1$, the measures $F$ and $G$ have the same zonoid if and only if $\int_{0}^{\infty} x \mathrm{~d} F(x)=\int_{0}^{\infty} x \mathrm{~d} G(x)$ and $\int_{-\infty}^{0} x \mathrm{~d} F(x)=\int_{-\infty}^{0} x \mathrm{~d} G(x)$. We give an example of two probability measures in $\mathbb{R}^{d}$ that have finite support.

Example 2.1. Let $F, G \in \mathscr{F}_{0}$, and assume that $F$ has support $x_{1}, \ldots, x_{n}$ and assigns probability $q_{i}$ to $x_{i}, i=1, \ldots, n$, and $G$ has support $y_{1}, \ldots, y_{m}$ with probabilities $r_{1}, \ldots, r_{m}$. Assume further that

$$
\sum_{\left\{x_{i} \mid \exists \lambda>0: x_{i}=\lambda p\right\}} q_{i} x_{i}=\sum_{\left\{y_{j} \mid \exists \lambda>0: y_{j}=\lambda\right.} r_{j} y_{j} .
$$

Equation (2.6) means that, for every $p$, the ray through $p$ contributes the same amount to the expectation of $F$ as to the expectation of $G$. It follows then that

$$
\int_{\{x:\langle x, p\rangle \geqslant 0\}} x \mathrm{~d} F(x)=\sum_{t} \sum_{\left\{x_{i}: \exists \lambda>0: x_{i}=\lambda t\right\}} q_{i} x_{i},
$$


where the first sum extends over all $t$ in the set $\left\{t: t \in S^{d-1} \cap\{t:\langle t, p\rangle \geqslant 0\}\right.$. Therefore (2.6) and Corollary 2.1 imply that $Z(F)=Z(G)$.

\section{The lift zonoid of a measure}

We introduce the notion of the lift zonoid of a measure in $\mathscr{L}$.

Definition 3.1. Let $F \in \mathscr{M l}$. For a measurable function $g: \mathbb{R}^{d} \rightarrow[0,1]$, consider the point

$$
z(F, g)=\left(\begin{array}{c}
z_{0}(F, g) \\
\zeta(F, g)
\end{array}\right) \in \mathbb{R}^{d+1}
$$

where

$$
z_{0}(F, g)=\int_{\mathbb{R}^{d}} g(x) \mathrm{d} F(x), \quad \zeta(F, g)=\int_{\mathbb{R}^{d}} g(x) x \mathrm{~d} F(x) .
$$

The set

$$
\hat{Z}(F) \equiv\left\{z(F, g): g: \mathbb{R}^{d} \rightarrow[0,1] \text { measurable }\right\}
$$

is called the lift zonoid of $F$.

The definition has the following geometric meaning. We add the coordinate $x_{0}$ and embed $\mathbb{R}^{d}$ as the hyperplane $H_{\alpha(F)}=\left\{x \in \mathbb{R}^{d+1}: x_{0}=\alpha(F)\right\}$ in $\mathbb{R}^{d+1}$. The given $d$-variate measure $F \in \mathscr{C l}$ is embedded as a measure on this hyperplane. The resulting measure $\hat{F}$ on $\mathbb{R}^{d+1}$ is called the lifted measure, and the lift zonoid of $F$ equals the zonoid of this. Formally

$$
\hat{Z}(F)=Z(\hat{F}), \quad \hat{F}(B)=F\left(\pi_{0}\left(B \cap H_{\alpha(F)}\right)\right),
$$

where $\pi_{0}$ is the projection on the last $d$ coordinates. From Definition 3.1 it immediately follows that $Z(F)=\pi_{0}(\hat{Z}(F))$, the projection of the lift zonoid on the last $d$ coordinates is the zonoid. For $F \in \mathscr{F}_{0}$ the lift zonoid is the expectation of the random segment

$$
\left[\mathbf{0},\left(\begin{array}{c}
1 \\
X
\end{array}\right)\right]
$$

where $X$ is a random vector distributed by $F$.

Another important special case arises if $F$ has finite support in $\mathbb{R}^{d}$. The lift zonoid in this case is a convex polytope which is called the lift zonotope of $F$.

Let $A=\left(a_{i k}\right)$ be a matrix in $\mathbb{R}^{n \times(d+1)}$ with non-negative first column. The first column is indexed with zero. Let $F_{A} \in \mathscr{C}_{0}$ be a measure that gives mass $a_{i 0}$ to the point $a_{i}=\left(a_{i 1}, \ldots, a_{i d}\right), i=1, \ldots, n$. Then the lift zonotope of $F_{A}$ is

$$
\hat{Z}\left(F_{A}\right)=\left\{\left(x_{0}, x\right)^{\mathrm{T}} \in \mathbb{R}^{d+1}: x_{0}=\sum_{i=1}^{n} g(i) a_{i 0}, x=\sum_{i=1}^{n} g(i) a_{i 0} \cdot a_{i}, 0 \leqslant g(i) \leqslant 1 \text { for all } i\right\} .
$$


A zonotope is a sum of line segments. In particular,

$$
\hat{Z}\left(F_{A}\right)=a_{10} \cdot\left[\mathbf{0},\left(1, a_{1}\right)^{\mathrm{T}}\right] \oplus \ldots \oplus a_{n 0} \cdot\left[\mathbf{0},\left(1, a_{n}\right)^{\mathrm{T}}\right] .
$$

The following lemma shows the shape of the lift zonoid in the case $d=1$.

Lemma 3.1. Let $d=1, F \in \mathscr{C}_{0}$.

(i) Then $\hat{Z}(F)$ is the convex hull of the following points in $\mathbb{R}^{d}$ : the origin, the point $(\alpha(F), \mu(F))^{\prime}$, and all points

$$
\left(\int_{]-\infty, y]} \mathrm{d} F(x), \int_{]_{-\infty, y]}} x \mathrm{~d} F(x)\right)^{\mathrm{T}}, \quad y \in \mathbb{R},
$$

and

$$
\left(\int_{[y,+\infty[} \mathrm{d} F(x), \int_{[y,+\infty[} x \mathrm{~d} F(x)\right)^{\mathrm{T}}, \quad y \in \mathbb{R} .
$$

(ii) Equivalently, $\hat{Z}(F)$ is the convex hull of

$$
\left(t \cdot \alpha(F), \int_{0}^{t} F^{-1}(s) \mathrm{d} s\right)^{\mathrm{T}}, \quad 0 \leqslant t \leqslant 1,
$$

and

$$
\left(t \cdot \alpha(F), \int_{1-t} F^{-1}(s) \mathrm{d} s\right)^{\mathrm{T}}, \quad 0 \leqslant t \leqslant 1,
$$

where $F^{-1}(s)=\inf \{x \in \mathbb{R}: F(x) \geqslant s\}$ is the usual inverse distribution function of $F$.

The proof of Lemma 3.1 follows from Theorem 2.1, and is left to the reader. Observe that the origin and the point $(\alpha(F), \mu(F))^{\mathrm{T}}$ can be represented as the above integrals (3.2) and (3.3) with $y=+\infty$ and $y=-\infty$, respectively: $(0,0)=\left(\int \varnothing \mathrm{d} F(x), \int \varnothing x \mathrm{~d} F(x)\right)$ and $(\alpha(F), \mu(F))=\left(\int_{]-\infty,+\infty[} \mathrm{d} F(x), \int_{]-\infty,+\infty[} x \mathrm{~d} F(x)\right)$.

Theorem 3.1. (i) The lift zonoid of $F \in \mathscr{C}$ is convex and contains $\mathbf{0} \in \mathbb{R}^{d+1}$.

(ii) If $F \in \mathscr{C l}_{0}$, the lift zonoid is compact and symmetric around

$$
\frac{1}{2}\left(\begin{array}{c}
\alpha(F) \\
\mu(F)
\end{array}\right)
$$

(iii) If the support of $F$ is in $\mathbb{R}_{+}^{d}$, then $\hat{Z}(F)$ is contained in the $(d+1)$-dimensional rectangle between $\mathbf{0}$ and

$$
\left(\begin{array}{l}
\alpha(F) \\
\mu(F)
\end{array}\right)
$$

These properties of the lift zonoid follow immediately from Proposition 2.1, Next we show that the lift zonoid is positive linear and continuous. 
Theorem 3.2. Let $F, F_{1}$ and $F_{2} \in \mathscr{C}_{0}, \beta>0$. Then

$$
\hat{Z}(\beta F)=\beta \hat{Z}(F), \quad \hat{Z}\left(F_{1}+F_{2}\right)=\hat{Z}\left(F_{1}\right) \oplus \hat{Z}\left(F_{2}\right) .
$$

Proof. Positive homogeneity, $\hat{Z}(\beta F)=\beta \hat{Z}(F)$, is immediate from Definition 2.1. To prove additivity, we recall that the support function of the Minkowski sum, $\hat{Z}\left(F_{1}\right) \oplus \hat{Z}\left(F_{2}\right)$, is equal to the sum of support functions of $\hat{Z}\left(F_{1}\right)$ and $\hat{Z}\left(F_{2}\right)$. Thus, we obtain

$$
\begin{aligned}
h\left(Z\left(\hat{F}_{1}\right) \oplus Z\left(\hat{F}_{2}\right), p\right) & =h\left(Z\left(\hat{F}_{1}\right), p\right)+h\left(Z\left(\hat{F}_{2}\right), p\right) \\
& =\int_{\{x:\langle x, p\rangle \geqslant 0\}}\langle x, p\rangle \mathrm{d} \hat{F}_{1}(x)+\int_{\{x:\langle x, p\rangle \geqslant 0\}}\langle x, p\rangle \mathrm{d} \hat{F}_{2}(x) \\
& =\int_{\{x:\langle x, p\rangle \geqslant 0\}}\langle x, p\rangle \mathrm{d}\left(\hat{F}_{1}(x)+\hat{F}_{2}(x)\right)=h\left(Z\left(\hat{F}_{1}+\hat{F}_{2}\right), p\right) \\
& =h\left(\hat{Z}\left(F_{1}+F_{2}\right), p\right) .
\end{aligned}
$$

Here we have used Theorem 2.1 and the fact that the adding and lifting of measures can be interchanged, hence $Z\left(\hat{F}_{1}+\hat{F}_{2}\right)=\hat{Z}\left(F_{1}+F_{2}\right)$. From the equality of support functions we conclude the equality of sets, hence (3.6).

Theorem 3.3. Let $F \in \mathscr{C}_{0}$, and let $\left(F_{n}\right)_{n \in \mathbb{N}}$ be a bounded sequence in $\mathscr{C}_{0}$. Then $\hat{Z}\left(F_{n}\right)$ converges to $\hat{Z}(F)$ in the Hausdorff distance if and only if $\left(F_{n}\right)_{n \in \mathbb{N}}$ converges weakly to $F$.

Proof. If the sequence $F_{n}$ converges weakly to $F$ and $F_{n} \leqslant G, G \in \mathscr{C}_{0}$, then it is easy to check that $\delta\left(\hat{Z}\left(F_{n}\right), \hat{Z}(F)\right) \rightarrow 0$, where $\delta$ is the Hausdorff distance (Bolker 1969).

To prove the reverse, we observe that the convergence of zonoids $\hat{Z}\left(F_{n}\right)$ to $\hat{Z}(F)$ in the Hausdorff metric implies uniform convergence of the support functions. That is, the integrals $\int \mathbb{R}^{d} \max \left\{0, p_{0}+\langle p, x\rangle\right\} \mathrm{d} F_{n}(x)$ converge, for $\left(p_{0}, p\right) \in S^{d}$, uniformly to $\int \mathbb{R}^{d} \max \left\{0, p_{0}+\langle p, x\rangle\right\} \mathrm{d} F(x)$. Then the derivatives in direction $p$ converge (the derivative in a direction is a uniform limit),

$$
\int_{\left\{x: p_{0}+\langle p, x\rangle \geqslant 0\right\}} \mathrm{d} F_{n}(x) \rightarrow \int_{\left\{x: p_{0}+\langle p, x\rangle \geqslant 0\right\}} \mathrm{d} F(x),
$$

and this holds for every $\left(p_{0}, p\right) \in \mathbb{R}^{d+1}$.

Given a probability measure $F \in \mathscr{F}$ and some $p \in \mathbb{R}^{d}$, we define

$$
F_{p}(t)=\int_{\left\{x \in \mathbb{R}^{d}:\langle x, p\rangle \leqslant t\right)} \mathrm{d} F(x), \quad t \in \mathbb{R} .
$$

If $F$ is the distribution of a random vector $X$, then $F_{p}$ is the distribution of the random variable $\langle X, p\rangle$.

Without loss of generality we assume that the above $F_{n}$ and $F$ are probability measures, 
since the convergence of zonoids $\hat{Z}\left(F_{n}\right)$ to $\hat{Z}(F)$ implies that $\lim \alpha\left(F_{n}\right)=\alpha(F)$. Now let $F_{n}$ be the probability distribution of $X_{n}$ and $F$ the probability distribution of $X$. For every $p \in S^{d-1}$, (3.7) yields weak convergence $\left(F_{n}\right)_{p} \rightarrow F_{p}$. By the Lévy continuity theorem (e.g., Laha and Rohatgi 1979), $\left(F_{n}\right)_{p} \rightarrow F_{p}$ implies convergence of the characteristic functions, $\mathrm{E}\left(\exp \left(\mathrm{i} t\left\langle X_{n}, p\right\rangle\right) \rightarrow \mathrm{E}\left(\exp (\mathrm{i} t\langle X, p\rangle), \quad t \in \mathbb{R} . \quad\right.\right.$ At $t=1$ we obtain $\mathrm{E}\left(\exp \left(\mathrm{i}\left\langle X_{n}, p\right\rangle\right)\right.$ $\rightarrow \mathrm{E}\left(\exp \left(\mathrm{i}\left\langle X_{n}, p\right\rangle\right)\right.$, for all $p \in \mathbb{R}^{d}$. Again by the Lévy theorem we conclude weak convergence $F_{n} \rightarrow F$.

Remark 3.1. In contrast to the zonoid, we prove at the end of this section, the lift zonoid uniquely determines the underlying measure. Hence the lift zonoid provides an embedding of the set of measures $\mathscr{L}_{0}$ into the space of convex compact sets in $\mathbb{R}^{d+1}$. The embedding is positive linear and, with respect to the weak bounded convergence of measures and the Hausdorff distance of compacts, a homoeomorphism.

The zonoid of a measure has properties analogous to those shown for the lift zonoid in Theorems 3.1 and 3.2. Also the zonoid $Z(F)$ is continuous on $F$. Since the underlying measure of a zonoid is not unique, the reverse direction of Theorem 3.3 fails. However, for even measures on the sphere the result holds; see Goodey and Weil (1993, p. 1301).

The inclusion of lift zonoids yields a useful ordering of measures; see Section 5 below. We characterize the ordering by the following theorem.

Theorem 3.4. Let $F, G \in \mathscr{C}_{0}$. Then $\hat{Z}(F) \subset \hat{Z}(G)$ if and only if $\hat{Z}\left(F_{p}\right) \subset \hat{Z}\left(G_{p}\right)$ for all $p \in \mathbb{R}^{d}$.

Proof. In view of (3.1) and Theorem 2.1, the support function of the lift zonoid is

$$
\begin{aligned}
h\left(\hat{Z}(F),\left(p_{0}, p\right)\right)=h\left(Z(\hat{F}),\left(p_{0}, p\right)\right) & =\int_{\mathbb{R}^{d+1}} \max \left\{0, x_{0} p_{0}+\langle x, p\rangle\right\} \mathrm{d} \hat{F}\left(x_{0}, x\right) \\
& =\int_{\mathbb{R}^{d}} \max \left\{0, p_{0}+\langle x, p\rangle\right\} \mathrm{d} F(x) \\
& =\int_{\mathbb{R}} \max \left\{0, p_{0}+t\right\} \mathrm{d} F_{p}(t) .
\end{aligned}
$$

Therefore, $\hat{Z}(F) \subset \hat{Z}(G)$ if and only if $h(Z(\hat{F}), \cdot) \leqslant h(Z(\hat{G}), \cdot)$ if and only if

$$
\left.\left.\int_{\mathbb{R}} \max \left\{0, p_{0}+t\right)\right\} \mathrm{d} F_{p}(t) \leqslant \int_{\mathbb{R}} \max \left\{0, p_{0}+t\right)\right\} \mathrm{d} G_{p}(t)
$$

for all $p_{0} \in \mathbb{R}, \quad p \in \mathbb{R}^{d}$. As in (3.8), the support function of $Z\left(\widehat{F_{p}}\right)$ is given by $h\left(Z\left(\widehat{F_{p}}\right),\left(q_{0}, q_{1}\right)\right)=\int_{\mathbb{R}} \max \left\{0, q_{0}+t q_{1}\right\} \mathrm{d} F_{p}(t)$, and similarly that of $Z\left(\widehat{G_{p}}\right)$. Therefore, (3.9) is equivalent to $h\left(Z\left(\hat{F}_{p}\right),\left(p_{0}, 1\right) \leqslant h\left(Z\left(\hat{G}_{p}\right),\left(p_{0}, 1\right)\right)\right.$. Because support functions are homogeneous, this is equivalent to $h\left(Z\left(\hat{F}_{p}\right), \cdot\right) \leqslant h\left(Z\left(\hat{G}_{p}\right), \cdot\right)$ for all $p \in \mathbb{R}^{d}$, hence to $\hat{Z}\left(F_{p}\right) \subset \hat{Z}\left(G_{p}\right)$ for all $p \in \mathbb{R}^{d}$. 
Remark 3.2. The lift zonoid $\hat{Z}\left(F_{p}\right)$ is, in fact, a two-dimensional projection of $\hat{Z}(F)$. For some fixed $p \in \mathbb{R}^{d}$, let $p r_{p}$ be the projection of $\mathbb{R}^{d+1}$ onto the two-dimensioned projection plane that is spanned by the points $(1,0, \ldots, 0)$ and $(0, p)$. Now, for $p_{0} \in \mathbb{R}$, consider the extreme point $z \in \hat{Z}(F)$ in the direction $\left(p_{0}, p\right)$. Its projection by $p r_{p}$ equals

$$
\left(\int_{\left\{p_{0}+\langle p, x\rangle \geqslant 0\right\}} \mathrm{d} F(x), \int_{\left\{p_{0}+\langle p, x\rangle \geqslant 0\right\}}\langle p, x\rangle \mathrm{d} F(x)\right)=\left(\int_{\left\{p_{0}+t \geqslant 0\right\}} \mathrm{d} F_{p}(t), \int_{\left\{p_{0}+t \geqslant 0\right\}} t \mathrm{~d} F_{p}(t)\right) .
$$

The latter is an extreme point of $\hat{Z}\left(F_{p}\right)$ and has the form (3.2) or (3.3) or equals $(0,0)$ or $\left(\alpha\left(F_{p}\right), \mu\left(F_{p}\right)\right)$. For every $p_{0}$, the point $\left(p_{0}, p\right)$ belongs to the projection plane. Therefore projections of other points of the lift zonoid belong to the convex hull of projections of such extreme points. According to Lemma 3.1 we obtain the result.

Theorem 3.5. Every measure $F \in \mathscr{C}_{0}$ is uniquely determined by its lift zonoid.

Proof. First, let $F, G \in \mathscr{F}_{0}$. Let $X, Y$ be random vectors that are distributed according to $F$ and $G$, respectively. Assume $\hat{Z}(F)=\hat{Z}(G)$. From Theorem 3.4 and Lemma 3.1 it follows that for every $p \in \mathbb{R}^{d}$

$$
\int_{0}^{t} F_{p}^{-1}(s) \mathrm{d} s=\int_{0}^{t} G_{p}^{-1}(s) \mathrm{d} s, \quad t \in[0,1],
$$

therefore $F_{p}^{-1}(t)=G_{p}^{-1}(t)$ for all $t$. We conclude that $F_{p}=G_{p}$, i.e., $\langle X, p\rangle$ is distributed like $\langle Y, p\rangle$, for every $p \in \mathbb{R}^{d}$. The Cramér-Wold theorem (e.g. Mardia et al. 1979) then yields that $X$ and $Y$ have the same distribution; hence the proposition for probability distributions.

Now, let $F, G \in \mathscr{C}_{0}$. Then $\hat{Z}(F)=\hat{Z}(G)$ implies that $\alpha(F)=\alpha(G)$ and $\hat{Z}\{(1 / \alpha(F)) F\}$ $=\hat{Z}\{(1 / \alpha(G)) G\}$. Because $(1 / \alpha(F)) F$ and $(1 / \alpha(G)) G \in \mathscr{F}_{0}$, we get $(1 / \alpha(F)) F=$ $(1 / \alpha(G)) G$ by the above, and therefore $F=G$.

Corollary 3.1. For every $F \in \mathscr{C}_{0}$ its lift zonoid is uniquely determined by the twodimensional projections $\operatorname{pr}_{p}(\hat{Z}(F)), p \in S^{d-1}$.

Proof. This follows from Theorems 3.5, 3.4 and Remark 3.2.

Our approach also yields a new metric among measures that is based on the Hausdorff distance.

Corollary 3.2. Let $d: \mathscr{C}_{0} \times \mathscr{C}_{0} \rightarrow \mathbb{R}_{+}$be a function of the form

$$
d\left(F_{1}, F_{2}\right)=\delta\left(\hat{Z}\left(F_{1}\right), \hat{Z}\left(F_{2}\right)\right), \quad F_{1}, F_{2} \in \mathscr{C l}_{0},
$$

where $\delta$ is the Hausdorff distance. Then $d$ is a metric in $\mathscr{M b}_{0}$. 
Proof. From Theorem 3.5 we see that $d\left(F_{1}, F_{2}\right)=0$ if and only if $F_{1}=F_{2}$. Symmetry and the triangle inequality follow from the same properties of the Hausdorff distance.

By the embedding property, the lift zonoid can be used in place of the measure. In fact, from a given lift zonoid, $\hat{Z}$, we can reconstruct the measure via the inversion of the Radon transform (Helgason 1980):

$$
\frac{\partial^{2} h\left(\hat{Z},\left(p_{0}, p\right)\right)}{\partial p_{0}^{2}}
$$

is the Radon transform of a measure at the point $\left(p_{0}, p\right)$. In what follows we illustrate several applications.

\section{Expected volume of a random convex hull}

We will exhibit a relation between the convex hull of a random sample in $d$-space and the lift zonoid of the probability measure from which they are sampled.

Beginning with the work of Rényi and Sulanke (1963; 1964) and Efron (1965), the convex hull of random points has been studied. Here we provide bounds on the expected volume in terms of the volume of the lift zonoid of the sampling distribution. The only restriction we impose is that the distribution has a finite expectation of the norm.

Definition 4.1. Random lift zonotope. Let $X_{1}, \ldots, X_{n}$ be $n$ independent random points in $\mathbb{R}^{d}$, each having the probability distribution $F \in \mathscr{F}_{0}$. The random lift zonotope of $X_{1}, \ldots, X_{n}$ is the random zonotope in $\mathbb{R}^{d+1}$ defined by

$$
\hat{Z}\left(X_{1}, \ldots, X_{n}\right)=\oplus_{i=1}^{n}\left[\mathbf{0}, \frac{1}{n}\left(1, X_{i}\right)\right] .
$$

The following theorem shows that the expected volume of the random lift zonotope equals, up to a constant, the volume of the lift zonoid of the random vector.

Theorem 4.1. Let $X_{1}, \ldots, X_{n}$ be independent random vectors each of which is distributed as F. Then

$$
\operatorname{EVol}\left(\hat{Z}\left(X_{1}, \ldots, X_{n}\right)\right)=\frac{(n-1) \cdots(n-d)}{n^{d}} \operatorname{Vol}(\hat{Z}(F))
$$

Proof. Let $M_{X}$ be a $d \times d$ matrix whose columns are independent and identically distributed as $F$. Due to Proposition 2.2 and Vitale (1991a), we have that $\operatorname{Vol}(Z(X))=(d !)^{-1} \mathrm{E}\left|\operatorname{det} M_{X}\right|$. The lift zonoid of $X$ is the zonoid of the random vector $\hat{X}=(1, X)$ in $\mathbb{R}^{d+1}$ that has 1 for sure as its first component. Thus the volume of the lift zonoid of $X$ equals

$$
\operatorname{Vol}(\hat{Z}(F))=\frac{1}{(d+1) !} \mathrm{E}\left|\operatorname{det} M_{\hat{X}}\right| \text {. }
$$


Given points $x_{1}, \ldots, x_{n}$ in $\mathbb{R}^{d+1}$, a zonotope $\oplus_{i=1}^{n}\left[0, x_{i}\right]$ is a convex polyhedron, whose volume equals (see Shephard 1974)

$$
\sum_{1 \leqslant i_{1}<\ldots<i_{d+1} \leqslant n}\left|\operatorname{det}\left(x_{i_{1}}, \ldots, x_{i_{d+1}}\right)\right| .
$$

In view of (4.4), we have

$$
\operatorname{Vol}\left(\hat{Z}\left(X_{1}, \ldots, X_{n}\right)\right)=\sum_{1 \leqslant i_{1}<\ldots<i_{d+1} \leqslant n}\left|\operatorname{det}\left(\frac{1}{n}\left(1, X_{i_{1}}\right), \ldots, \frac{1}{n}\left(1, X_{i_{d+1}}\right)\right)\right| .
$$

Therefore, with respect to (4.3) and (4.5), we obtain

$$
\begin{aligned}
& \operatorname{E} \operatorname{Vol}\left(\hat{Z}\left(X_{1}, \ldots, X_{n}\right)\right)=\sum_{1 \leqslant i_{1}<\ldots<i_{d+1} \leqslant n} \mathrm{E}\left|\operatorname{det}\left(\frac{1}{n}\left(1, X_{i_{1}}\right), \ldots, \frac{1}{n}\left(1, X_{i_{d+1}}\right)\right)\right| \\
& =\frac{1}{n^{d+1}}(d+1) ! \sum_{1 \leqslant i_{1}<\ldots<i_{d+1} \leqslant n} \operatorname{Vol}(\hat{Z}(F))=\frac{1}{n^{d+1}}(d+1) !\left(\begin{array}{c}
n \\
d+1
\end{array}\right) \operatorname{Vol}(\hat{Z}(F)) \\
& =\operatorname{Vol}(\hat{Z}(F)) \prod_{i=1}^{d} \frac{n-i}{n}
\end{aligned}
$$

and the proof is complete.

Let $x_{1}, \ldots, x_{n}$ be points in $\mathbb{R}^{d}$, and consider the zonotope

$$
Z_{n}=\left[\mathbf{0}, \frac{1}{n}\left(1, x_{1}\right)\right] \oplus \ldots \oplus\left[\mathbf{0}, \frac{1}{n}\left(1, x_{n}\right)\right] .
$$

We want to establish inequalities between the volume of $Z_{n}$ and the volume of the convex hull, $C_{n}=\operatorname{conv}\left\{x_{1}, \ldots, x_{n}\right\}$. While $Z_{n}$ is a set in $\mathbb{R}^{d+1}, C_{n}$ is one in $\mathbb{R}^{d}$. In what follows, the volumes are defined with respect to these dimensions. We first provide a lower bound for the volume of $C_{n}$.

\section{Theorem 4.2.}

$$
\operatorname{Vol}\left(C_{n}\right) \geqslant 2^{d}(d+1) \operatorname{Vol}\left(Z_{n}\right)
$$

Proof. Consider the hyperplanes $H_{\alpha}=\left\{x \in \mathbb{R}^{d+1} \mid x_{0}=\alpha\right\}$ and the half-spaces $H_{\leqslant \alpha}=$ $\left\{x \in \mathbb{R}^{d+1} \mid x_{0} \leqslant \alpha\right\} \quad$ and $\quad H_{\geqslant \alpha}=\left\{x \in \mathbb{R}^{d+1} \mid x_{0} \geqslant \alpha\right\}$. Let $S_{n}=Z_{n} \cap H_{1 / n}$. Then $C_{n}=n \cdot \pi_{0}\left(S_{n}\right)$, where $\pi_{0}$ is the projection on the last $d$ coordinates. Therefore the $d$ variate volume of $S_{n}$ equals

$$
\operatorname{Vol}\left(S_{n}\right)=\frac{1}{n^{d}} \operatorname{Vol}\left(C_{n}\right)
$$


It is easy to check that for any $\alpha \leqslant 1 / n$,

$$
n \cdot S_{n}=n \cdot\left(Z_{n} \cap H_{1 / n}\right)=\frac{1}{\alpha} \cdot\left(Z_{n} \cap H_{\alpha}\right) .
$$

Therefore, $Z_{n} \subset$ Cone $\left(S_{n}\right)$, where $\operatorname{Cone}(S)=\left\{\lambda s \mid \lambda \in \mathbb{R}_{+}, s \in S\right\}$ for $S \subset \mathbb{R}^{k}$. Because $Z_{n}$ is centrally symmetric around $\frac{1}{2}(1, \mu)$,

$$
\operatorname{Vol}\left(Z_{n}\right) \leqslant 2 \cdot \operatorname{Vol}\left(\operatorname{Cone}\left(S_{n}\right) \cap H_{\leqslant 1 / 2}\right) \text {. }
$$

Further, Cone $\left(S_{n}\right) \cap H_{\leqslant 1 / 2}$ is a pyramid in $\mathbb{R}^{d+1}$ that has base $\operatorname{Cone}\left(S_{n}\right) \cap H_{1 / 2}$ and height $1 / 2$. As the $d$-variate volume of the base amounts to $(n / 2)^{d}$, we obtain

$$
\operatorname{Vol}\left(\operatorname{Cone}\left(S_{n}\right) \cap H_{\leqslant 1 / 2}\right)=\frac{1}{d+1} \cdot \frac{1}{2} \cdot\left(\frac{n}{2}\right)^{d} \operatorname{Vol}\left(S_{n}\right) .
$$

In view of (4.8), (4.10), (4.11) we have

$$
\operatorname{Vol}\left(Z_{n}\right) \leqslant\left(\frac{n}{2}\right)^{d} \cdot \frac{1}{d+1} \cdot\left(\frac{1}{n}\right)^{d} \operatorname{Vol}\left(C_{n}\right)
$$

as required.

A reverse bound is established in the following theorem.

Theorem 4.3. Let $n \geqslant d+1$. Then

$$
\operatorname{Vol}\left(C_{n}\right) \leqslant \frac{n^{d+1}(d+1)}{n(d+1)-2 d} \operatorname{Vol}\left(Z_{n}\right)
$$

Proof. First, note that (4.13) can be written

$$
\operatorname{Vol}\left(Z_{n}\right) \geqslant \frac{1}{n^{d}}\left(\frac{n-2}{n}+2 \frac{1}{(d+1) n}\right) \operatorname{Vol}\left(C_{n}\right) .
$$

Consider the partition of $Z_{n}$ that consists of the two pyramids $S_{n}=Z_{n} \cap H_{\leqslant 1 / n}, \bar{S}_{n}=$ $Z_{n} \cap H_{\geqslant(n-1) / n}$ and the convex body $B_{n}=Z_{n} \cap H_{\leqslant(n-1) / n} \cap H_{\geqslant 1 / n}$. The pyramids have the same volumes of bases, $n^{-d} \operatorname{Vol}\left(C_{n}\right)$, and the same heights $1 / n$. So, their volumes sum to

$$
\frac{2}{n^{d}} \frac{1}{(d+1) n} \operatorname{Vol}\left(C_{n}\right) \text {. }
$$

The body $B_{n}$ is convex and has centrally, around the point $\frac{1}{2}(1, \mu)$, symmetric bases, $S_{n}$ and $\bar{S}_{n}$, and height $(n-2) / n$. Therefore, due to Schwartz symmetrization (Bonnesen and Fenchel 1934),

$$
\operatorname{Vol}\left(B_{n}\right) \geqslant \frac{n-2}{n} \operatorname{Vol}\left(S_{n}\right)=\frac{n-2}{n} \frac{1}{n^{d}} \operatorname{Vol}\left(C_{n}\right),
$$

which yields the proof. 
As a consequence of the above three theorems we have the following.

Theorem 4.4. Let $X_{1}, \ldots, X_{n}$ be random vectors independent and identically distributed as $F, n \geqslant d+1$. Then

$$
\begin{aligned}
\left(\frac{n}{2}\right)^{-d}\left(\prod_{i=1}^{d}(n-i)\right)(d+1) \operatorname{Vol}(\hat{Z}(F)) & \leqslant \operatorname{EVol}\left(\operatorname{conv}\left\{X_{1}, \ldots, X_{n}\right\}\right) \\
& \leqslant \frac{(d+1) n}{n(d+1)-2 d}\left(\prod_{i=1}^{d}(n-i)\right) \operatorname{Vol}(\hat{Z}(F)) .
\end{aligned}
$$

\section{Lift zonoids and variability of random vectors}

We propose a new ordering between random vectors in $\mathbb{R}^{d}$ that is weaker than dilation and has nice geometric properties. Our idea is to replace a probability distribution on $\mathbb{R}^{d}$ by its lift zonoid and to consider the order between random vectors that is induced by the inclusion of their lift zonoids.

\subsection{The lift zonoid order}

The zonoid order $\leqslant_{Z}$ between measures $F$ and $G \in \mathscr{L} b$ is defined by

$$
F \leqslant_{Z} G \text { if } Z(F) \subset Z(G) .
$$

Corollary 2.1 and Example 2.1 have shown that there exist many probability measures that are equivalent with respect to the zonoid order. The following definition avoids this drawback.

Definition 5.1. For $F, G \in \mathscr{C}_{0}$, we introduce the lift zonoid order $\leqslant_{L Z}$,

$$
F \leqslant_{L Z} G \text { if } \hat{Z}(F) \subset \hat{Z}(G) \text {. }
$$

It follows immediately from this definition and Theorem 3.1(ii) that $F \preccurlyeq_{L Z} G$ implies $\alpha(F) \leqslant \alpha(G)$ and $\mu(F) \leqslant \mu(G) . \leqslant_{L Z}$ is a partial order (reflexive, transitive and antisymmetric) on $\mathscr{C l}_{0}$.

Let $\mathscr{C}_{0}$ be the set of all random variables that have values in $\mathbb{R}^{d}$ and finite expectations, and, given $X, Y \in \mathscr{C}_{0}$, write $F_{X}, F_{Y} \in \mathscr{F}_{0}$ for their distributions. By defining

$$
X \leqslant_{L Z} Y \text { if } F_{X} \leqslant_{L Z} F_{Y},
$$

we obtain a preorder (reflexive and transitive) on $\mathscr{C}_{0}$. This induces a partial order on the factor space $\mathscr{C}_{0} /=_{\mathrm{d}}$. In view of Proposition 2.2 the preorder between $X$ and $Y, X \leqslant_{L Z} Y$, can be interpreted in the way that the 'set-valued expectation' of $X$ is 'smaller' than that of $Y$, $\mathrm{E}([\mathbf{0},(1, X)]) \subset \mathrm{E}([\mathbf{0},(1, Y)])$. The lift zonoid order ranks random vectors by their variability. 
In the remainder of this paper we investigate the properties of $\leqslant_{L Z}$ on $\mathscr{F}_{0}$ and on $\mathscr{C}_{0}$. We start with a well-known theorem and the definition of the dilation order. See Mosler and Scarsini (1991), Shaked and Shanthikumar (1994).

Theorem 5.1. Let $X, Y \in \mathscr{C}_{0}$. The following three conditions are equivalent.

(i) $\mathrm{E}[\phi(X)] \leqslant \mathrm{E}[\phi(Y)]$ for all convex $\phi: \mathbb{R}^{d} \rightarrow \mathbb{R}$ for which the expectations exist.

(ii) $\mathrm{E}[X]=\mathrm{E}[Y]$, and $\mathrm{E}[\phi(X)] \leqslant \mathrm{E}[\phi(Y)]$ for all increasing convex $\phi: \mathbb{R}^{d} \rightarrow \mathbb{R}$ for which the expectations exist.

(iii) $Y={ }_{\mathrm{d}} X+U$ with some $U$ for which $\mathrm{E}[U \mid X]=0$.

If one, and hence all, conditions of Theorem 5.1 are satisfied, $Y$ is called a dilation of $X$, $X \preccurlyeq_{\text {dil }} Y$. For the lift zonoid order we state the following characterization theorem. A function $\phi: \mathbb{R}^{d} \rightarrow \mathbb{R}$ is called convex-linear if $\phi=\theta \circ l$ with some linear $l: \mathbb{R}^{d} \rightarrow \mathbb{R}$ and some convex $\theta: \mathbb{R} \rightarrow \mathbb{R}$.

Theorem 5.2. The following five conditions are equivalent.

(i) $X \leqslant_{L Z} Y$.

(ii) $\mathrm{E}[\phi(X)] \leqslant \mathrm{E}[\phi(Y)]$ holds for every convex-linear function $\phi$ for which both expectations exist.

(iii) For all $p \in \mathbb{R}^{d},\langle X, p\rangle \leqslant_{L Z}\langle Y, p\rangle$.

(iv) For all $p \in \mathbb{R}^{d},\langle Y, p\rangle={ }_{\mathrm{d}}\langle X, p\rangle+U_{p}$, where $U_{p}$ is a random vector in $\mathbb{R}^{d}$ and $\mathrm{E}\left[U_{p} \mid\langle X, p\rangle\right]=0$.

(v) For all $p \in \mathbb{R}^{d}$ and all $t \in[0,1], \int_{0}^{t} F_{p}^{-1}(s) \mathrm{d} s \geqslant \int_{0}^{t} G_{p}^{-1}(s) \mathrm{d} s$.

Corollary 5.1. $X \leqslant_{\text {dil }} Y \Rightarrow X \leqslant_{L Z} Y$.

As every convex-linear function is convex, the corollary is obvious from Theorems 5.1(i) and 5.2(ii).

If $d>1$, the reverse implication, $X \leqslant_{L Z} Y \Rightarrow X \leqslant_{\text {dil }} Y$, does not hold in general (Koshevoy 1995). But there are cases under which the two orders coincide. See Koshevoy (1995) and Corollary 5.3 below.

Proof of Theorem 5.2. By Theorem 3.4, $X \leqslant_{L Z} Y$ if and only if $\hat{Z}\left(F_{p}\right) \subset \hat{Z}\left(G_{p}\right)$ for all $p \in \mathbb{R}^{d} . F_{p}$ and $G_{p}$ are the distributions of $\langle X, p\rangle$ and $\langle Y, p\rangle$, respectively. Hence (i) $\Leftrightarrow$ (iii).

From Lemma 3.1(ii) it follows that, for $d=1$ and $H \in \mathscr{F}_{0}$, the border of $\hat{Z}(H)$ consists of the two curves

$$
\left(t, \int_{0}^{t} H^{-1}(s) \mathrm{d} s\right)^{\mathrm{T}},\left(t, \int_{1-t}^{1} H^{-1}(s) \mathrm{d} s\right)^{\mathrm{T}}, \quad 0 \leqslant t \leqslant 1 .
$$


Therefore $\langle X, p\rangle \preccurlyeq_{L Z}\langle Y, p\rangle$ if and only if

$$
\begin{gathered}
\int_{0}^{t} F_{p}^{-1}(s) \mathrm{d} s \geqslant \int_{0}^{t} G_{p}^{-1}(s) \mathrm{d} s \quad \forall t \in[0,1] \\
\mathrm{E}[\langle X, p\rangle]-\int_{0}^{t} F_{p}^{-1}(s) \mathrm{d} s \leqslant \mathrm{E}[\langle Y, p\rangle]-\int_{0}^{t} G_{p}^{-1}(s) \mathrm{d} s \quad \forall t \in[0,1] .
\end{gathered}
$$

This shows that (iii) implies (5.2) for all $p$, hence (v). On the other hand, for every $p \in \mathbb{R}^{d}$, we see from (5.2) that $\mathrm{E}[\langle X, p\rangle] \leqslant \mathrm{E}[\langle Y, p\rangle]$. The same holds for $-p$. Therefore $\mathrm{E}[\langle X, p\rangle]=\mathrm{E}[\langle Y, p\rangle]$ for all $p$. It follows that (5.2) for all $p$ implies (5.3) for all $p$, and therefore (v) implies (iii).

Because the expectations are equal, (v) means that, for all $p, G_{p}$ is a dilation of $F_{p}$. The equivalences (v) $\Leftrightarrow$ (iv) and (v) $\Leftrightarrow$ (ii) then follow from Theorem 5.1.

Let $l: \mathbb{R}^{d} \rightarrow \mathbb{R}$ be linear and $\theta: \mathbb{R} \rightarrow \mathbb{R}$ be convex. Then $\phi=\theta \circ l$ is a convex function $\mathbb{R}^{d} \rightarrow \mathbb{R}$.

The dilation order is positive homogeneous, i.e., $X \leqslant_{\text {dil }} Y$ implies that $\alpha X \leqslant_{\text {dil }} \alpha Y$ for every $\alpha>0$, and the same holds for the lift zonoid order. More general, both the dilation order and the lift zonoid order are preserved under affine transformations.

Proposition 5.1. Let $A$ be a $k \times d$ matrix, and $\beta \in \mathbb{R}^{k}$. Then

(i) $X \preccurlyeq_{\text {dil }} Y \Rightarrow A X+\beta \leqslant_{\text {dil }} A Y+\beta$.

(ii) $X \leqslant_{L Z} Y \Rightarrow A X+\beta \leqslant_{L Z} A Y+\beta$.

Proof. The proposition is a consequence of Theorem 5.2(ii) and Theorem 5.1(i).

In particular, if two random vectors are lift zonoid ordered then all their marginals are ordered as well. It follows from Theorem 3.3 that the lift zonoid order is continuous in the following sense.

Theorem 5.3. Let $F, G \in \mathscr{F}_{0}$, and consider a sequence $\left(G_{n}\right)_{n \in \mathbb{N}}$ bounded in $\mathscr{C l}_{0}$ that converges weakly to $G$. Then

(i) $F \preccurlyeq_{L Z} G_{n} \forall n \in \mathbb{N} \Rightarrow F \leqslant_{L Z} G$.

(ii) $G_{n} \preccurlyeq_{L Z} F \forall n \in \mathbb{N} \Rightarrow G \preccurlyeq_{L Z} F$.

\subsection{The lift zonoid of a marginal distribution}

We show that the lift zonoid of a marginal distribution is the proper projection of the lift zonoid. From this again it is seen that the lift zonoid ordering of two random vectors implies the same for all their marginals. Under stochastic independence a reverse result is proved.

Now, for $x \in \mathbb{R}^{d}$ and $J \subset\{1,2, \ldots, d\}$, let $x_{J}$ denote the vector of components $x_{i}, i \in J$, and $x_{-J}$ the vector of components $x_{i}, i \notin J$. If $F$ is the distribution of a random vector $X$ in $\mathbb{R}^{d}, F^{J}$ denotes the marginal distribution of $X_{J}$. 
Theorem 5.4. Let $F \in \mathscr{F}_{0}, J \subset\{1,2, \ldots, d\}$, and denotes $\mathrm{pr}_{J}:\left(x_{0}, x\right) \mapsto\left(x_{0}, x_{J}\right)$ for every $\left(x_{0}, x\right) \in \mathbb{R}^{d+1}$. Then $\operatorname{pr}_{J}(\hat{Z}(F))=\hat{Z}\left(F^{J}\right)$.

Proof. Extreme points of $\hat{X}\left(F^{J}\right)$ are of the form

$$
\left(\int_{\left\{x_{J}: p_{0}+\left\langle x_{J}, p_{J}\right\rangle \geqslant 0\right\}} \mathrm{d} F^{J}\left(x_{J}\right), \int_{\left\{x_{J}: p_{0}+\left\langle x_{J}, p_{J}\right\rangle \geqslant 0\right\}} x_{J} \mathrm{~d} F^{J}\left(x_{J}\right)\right) .
$$

Consider the projection of an extreme point of $\hat{Z}(F)$ in direction $\left(p_{0}, p_{J}, 0_{-J}\right)$ :

$$
\begin{aligned}
& \operatorname{pr}_{J}\left(\left(\int_{\left\{x: p_{0}+\left\langle x, p_{J}\right\rangle \geqslant 0\right\}} \mathrm{d} F(x), \int_{\left\{x: p_{0}+\left\langle x, p_{J}\right\rangle \geqslant 0\right\}} x \mathrm{~d} F(x)\right)\right) \\
& =\operatorname{pr}_{J}\left(\left(\int_{\left\{x^{J}: p_{0}+\left\langle x^{J}, p_{J}\right\rangle \geqslant 0\right\}} \mathrm{d} F^{J}\left(x^{J}\right), \int_{\left\{x^{J}: p_{0}+\left\langle x^{J}, p_{J}\right\rangle \geqslant 0\right\}} x \mathrm{~d} F^{J}\left(x^{J}\right)\right)\right) \\
& =\left(\int_{\left\{x_{J}: p_{0}+\left\langle x_{J}, p_{J}\right\rangle \geqslant 0\right\}} \mathrm{d} F^{J}\left(x_{J}\right), \int_{\left\{x_{J}: p_{0}+\left\langle x_{J}, p_{J}\right\rangle \geqslant 0\right\}} x_{J} \mathrm{~d} F^{J}\left(x_{J}\right)\right) .
\end{aligned}
$$

The projections of other points of $\hat{Z}(F)$ belong to the convex hull of points (5.5), yielding $\operatorname{pr}_{J}(\hat{Z}(F))=\hat{Z}\left(F^{J}\right)$.

Theorem 5.4 says that the lift zonoid of a marginal distribution $F^{J}$ is equal to the projection of the lift zonoid of $F$. This implies that the lift zonoid order between two distributions implies the lift zonoid order between all their marginals. The reverse is not true, but the following theorem holds.

Theorem 5.5. For $F, G \in \mathscr{F}_{0}$ and $J \subset\{1, \ldots, d\}$,

$$
\hat{Z}\left(F^{J} \cdot F^{-J}\right) \subset \hat{Z}\left(G^{J} \cdot G^{-J}\right) \Leftrightarrow \hat{Z}\left(F^{J}\right) \subset \hat{Z}\left(G^{J}\right) \text { and } \hat{Z}\left(F^{-J}\right) \subset \hat{Z}\left(G^{-J}\right) .
$$

Proof. The 'only if' part follows from Theorem 5.4. To prove the reverse, we consider the support function of $\hat{Z}\left(F^{J} \cdot F^{-J}\right)$. For given $\left(p_{0}, p\right) \in \mathbb{R}^{d}$,

$$
\begin{aligned}
h\left(\hat{Z}\left(F^{J} \cdot F^{-J}\right),\left(p_{0}, p\right)\right) & =\int_{\left\{x: p_{0}+\langle x, p\rangle \geqslant 0\right\}}\left(p_{0}+\langle x, p\rangle\right) \mathrm{d} F^{J}\left(x_{J}\right) \mathrm{d} F^{-J}\left(x_{-J}\right) \\
& =\int_{-\infty}^{\infty}\left[\int_{\left\{x_{-J}: p_{0}+\left\langle x_{-J}, p_{-J}\right\rangle=s\right\}} \psi\left(s, F^{J}\right) \mathrm{d} F^{-J}\left(x_{-J}\right)\right] \mathrm{d} s \\
& =\int_{\mathbb{R}^{-J}} \psi\left(p_{0}+\left\langle x_{-J}, p_{-J}\right\rangle, F^{J}\right) \mathrm{d} F^{-J}\left(x_{-J}\right),
\end{aligned}
$$

where we have introduced

$$
\psi\left(s, F^{J}\right)=\int_{\left\{x_{J}: s+\left\langle p_{J}, x_{J}\right\rangle \geqslant 0\right\}}\left(s+\left\langle p_{J}, x_{J}\right\rangle\right) \mathrm{d} F^{J}\left(x_{J}\right) .
$$


Note that $s \mapsto \psi\left(s, F^{J}\right)$ is a convex function, and $x_{-J} \mapsto \psi\left(p_{0}+\left\langle x_{-J}, p_{-J}\right\rangle, F^{J}\right)$ is a convex-linear function. Therefore, from $\hat{Z}\left(F^{-J}\right) \subset \hat{Z}\left(G^{-J}\right)$, Theorems 5.2(ii) and 5.6 we obtain

$$
\begin{aligned}
h\left(\hat{Z}\left(F^{J} \cdot F^{-J}\right),\left(p_{0}, p\right)\right) & =\int_{\mathbb{R}^{-J}} \psi\left(p_{0}+\left\langle x_{-J}, p_{-J}\right\rangle, F^{J}\right) \mathrm{d} F^{-J}\left(x_{-J}\right) \\
& \leqslant \int_{\mathbb{R}^{-J}} \psi\left(p_{0}+\left\langle x_{-J}, p_{-J}\right\rangle, F^{J}\right) \mathrm{d} G^{-J}\left(x_{-J}\right) \\
& =\int_{-\infty}^{\infty}\left[\int_{\left\{x_{-J}: p_{0}+\left\langle x_{-J}, p_{-J}\right\rangle=s\right\}} \psi\left(s, F^{J}\right) \mathrm{d} G^{-J}\left(x_{-J}\right)\right] \mathrm{d} s .
\end{aligned}
$$

Further, $\hat{Z}\left(F^{J}\right) \subset \hat{Z}\left(G^{J}\right)$ with Theorem 5.2(ii) yields $\psi\left(s, F^{J}\right) \leqslant \psi\left(s, G^{J}\right)$ for all $s$. Therefore the right-hand side of (5.7) is bounded by

$$
\int_{-\infty}^{\infty}\left[\int_{\left\{x_{-J}: p_{0}+\left\langle x_{-J}, p_{-J}\right\rangle=s\right\}} \psi\left(s, G^{J}\right) \mathrm{d} G^{-J}\left(x_{-J}\right)\right] \mathrm{d} s=h\left(\hat{Z}\left(G^{J} \cdot G^{-J}\right),\left(p_{0}, p\right)\right) .
$$

We conclude $h\left(\hat{Z}\left(F^{J} \cdot F^{-J}\right), \cdot\right) \leqslant h\left(\hat{Z}\left(G^{J} \cdot G^{-J}\right), \cdot\right)$, hence $\hat{Z}\left(F^{J} \cdot F^{-J}\right) \subset \hat{Z}\left(G^{J} \cdot G^{-J}\right)$.

Corollary 5.2. Let $X, Y, U, V \in \mathscr{D}_{0}$. Assume that $X$ and $Y$ are independent, $U$ and $V$ are independent, $X \preccurlyeq_{L Z} U, Y \preccurlyeq_{L Z} V$. Then, for every $\alpha \in[0,1]$,

$$
\alpha X+(1-\alpha) Y \leqslant_{L Z} \alpha U+(1-\alpha) V
$$

Proof. Consider the random vectors $\left(X^{\mathrm{T}}, Y^{\mathrm{T}}\right)^{\mathrm{T}}$ and $\left(U^{\mathrm{T}}, V^{\mathrm{T}}\right)^{\mathrm{T}}$ in $\mathbb{R}^{2 d}$. From the assumptions of Corollary 5.2 and Theorem 5.5 it follows that $\left(X^{\mathrm{T}}, Y^{\mathrm{T}}\right)^{\mathrm{T}} \leqslant_{L Z}\left(U^{\mathrm{T}}, V^{\mathrm{T}}\right)^{\mathrm{T}}$. Let $I_{d}$ denote the $d \times d$ unit matrix, $O_{d}$ denote the $d \times d$ zero matrix, and $A=\alpha\left(I_{d}, O_{d}\right)+$ $(1-\alpha)\left(O_{d}, I_{d}\right)$. Then $A\left(X^{\prime}, Y^{\prime}\right)^{\prime}=\alpha X+(1-\alpha) Y$ and $A\left(U^{\prime}, V^{\prime}\right)^{\prime}=\alpha U+(1-\alpha) V$. Proposition 5.1(ii) yields the assertion.

The next corollary says that, for vectors of stochastically independent components, the lift zonoid order is equivalent to the dilation order.

Corollary 5.3. Let $F, \quad G \in \mathscr{F}_{0}, \quad F(x)=\prod_{j=1}^{d} F^{j}\left(x_{j}\right), \quad G(x)=\prod_{j=1}^{d} G^{j}\left(x_{j}\right)$, and let $X=$ $\left(X_{1}, \ldots, X_{d}\right)^{\mathrm{T}}$ be distributed according to $F, Y=\left(Y_{1}, \ldots, Y_{d}\right)^{\mathrm{T}}$ according to $G$. Then

$$
X \preccurlyeq_{L Z} Y \Leftrightarrow X_{j} \preccurlyeq_{L Z} Y_{j}, \quad \forall j \Leftrightarrow X \preccurlyeq_{\mathrm{dil}} Y .
$$

Proof. By repeated application of Theorem 5.5, $F \preccurlyeq_{L Z} G$ if and only if $F^{j} \preccurlyeq_{L Z} G^{j}$ for all $j$. For univariate probability distributions, dilation and lift zonoid order coincide. Again, under the stochastic independence assumption, dilation of all univariate marginals is equivalent to $Y$ being a dilation of $X$. 


\subsection{Probability mixtures and convex convolutions}

Next, we show that the ordering $\preccurlyeq_{L Z}$ is preserved under mixtures of probability distributions.

Theorem 5.6. Let $F_{1}, F_{2}, G_{1}, G_{2}, H \in \mathscr{F}_{0}$. For every $\alpha \in[0,1]$, the following statements hold:

(i) If $F_{1} \preccurlyeq_{L Z} G_{1}$ and $F_{2} \preccurlyeq_{L Z} G_{2}$, then $\alpha F_{1}+(1-\alpha) F_{2} \preccurlyeq_{L Z} \alpha G_{1}+(1-\alpha) G_{2}$.

(ii) If $F_{1} \preccurlyeq_{L Z} H$ and $F_{2} \preccurlyeq_{L Z} H$, then $\alpha F_{1}+(1-\alpha) F_{2} \preccurlyeq_{L Z} H$.

(iii) If $H \preccurlyeq_{L Z} G_{1}$ and $H \preccurlyeq_{L Z} G_{2}$, then $H \preccurlyeq_{L Z} \alpha G_{1}+(1-\alpha) G_{2}$.

Proof. Obviously, (ii) and (iii) follow from (i). By assumption in (i), we have $\hat{Z}\left(F_{1}\right) \subset \hat{Z}\left(G_{1}\right)$ and $\hat{Z}\left(F_{2}\right) \subset \hat{Z}\left(G_{2}\right)$. From Theorem 3.2 it follows that for every $F_{1}, F_{2} \in \mathscr{F}_{0}$,

$$
\hat{Z}\left(\alpha F_{1}+(1-\alpha) F_{2}\right)=\alpha \hat{Z}\left(F_{1}\right) \oplus(1-\alpha) \hat{Z}\left(F_{2}\right) .
$$

Then $\hat{Z}\left(\alpha F_{1}+(1-\alpha) F_{2}\right) \subset \hat{Z}\left(\alpha G_{1}+(1-\alpha) G_{2}\right)$ holds, and we conclude (i).

The next proposition is about convex convolutions, i.e., convex combinations of independent random vectors.

Theorem 5.7. Let $X, Y, W \in \mathscr{C}_{0}, \alpha \in[0,1]$, and assume that $X$ and $Y$ are independent. Denote by $V$ the $\alpha$-mixture, with $F_{V}=\alpha F_{X}+(1-\alpha) F_{Y}$, and by $U$ the $\alpha$-convolution, $U=\alpha X+(1-\alpha) Y$, of $X$ and $Y$. Then the following statements hold:

(i) $U \leqslant_{L Z} V$.

(ii) If $X \leqslant_{L Z} W$ and $Y \leqslant_{L Z} W$ then $U \leqslant_{L Z} W$.

(iii) If $W \preccurlyeq_{L Z} X$ and $W \preccurlyeq_{L Z} Y$ then $W \preccurlyeq_{L Z} U$.

Theorem 5.7(ii) implies that, if a set of independent random vectors is $\leqslant_{L Z}$-smaller than a given random vector, then every convex combination of such vectors is smaller than the given vector, too. (iii) implies the same for a set of independent vectors that are $\leqslant_{L Z}$-greater than a given vector.

Proof. Let $F_{X}, F_{Y}, F_{U}, F_{V}$ and $F_{W}$ be the distributions of $X, Y, U, V$ and $W$, respectively. Show that, for $0 \leqslant \alpha \leqslant 1$, the inclusion

$$
\hat{Z}\left(F_{U}\right) \subset \alpha \hat{Z}\left(F_{X}\right) \oplus(1-\alpha) \hat{Z}\left(F_{Y}\right)
$$

holds. Let $z \in \hat{Z}\left(F_{U}\right)$. Then, with some $g: \mathbb{R}^{d} \rightarrow[0,1]$, 


$$
\begin{aligned}
z= & \int_{\mathbb{R}^{d}} g(u)(1, u)^{\mathrm{T}} \mathrm{d} F_{U}(u) \\
= & \int_{\mathbb{R}^{d}} \int_{\mathbb{R}^{d}} g(\alpha x+(1-\alpha) y)(1, \alpha x+(1-\alpha) y)^{\mathrm{T}} \mathrm{d} F_{X}(x) \mathrm{d} F_{Y}(y) \\
= & \alpha \int_{\mathbb{R}^{d}}\left[\int_{\mathbb{R}^{d}} g(\alpha x+(1-\alpha) y)(1, x)^{\mathrm{T}} \mathrm{d} F_{X}(x)\right] \mathrm{d} F_{Y}(y) \\
& +(1-\alpha) \int_{\mathbb{R}^{d}}\left[\int_{\mathbb{R}^{d}} g(\alpha x+(1-\alpha) y)(1, y)^{\mathrm{T}} \mathrm{d} F_{Y}(y)\right] \mathrm{d} F_{X}(x) .
\end{aligned}
$$

The inner integral of the first summand in (5.11) is an element of $\hat{Z}\left(F_{X}\right)$ for every $y$. Integration over $y$ again yields an element of $\hat{Z}\left(F_{X}\right)$ because $\hat{Z}\left(F_{X}\right)$ is convex. Therefore the first summand in (5.11) equals $\alpha z^{*}$ with some $z^{*} \in \hat{Z}\left(F_{X}\right)$. Similarly, the second summand amounts to $(1-\alpha) z^{* *}$, where $z^{* *} \in \hat{Z}\left(F_{Y}\right)$. We conclude (5.10). Therefore, $\hat{Z}\left(F_{U}\right) \subset$ $\hat{Z}\left(F_{V}\right)$, which proves part (i) of the theorem.

From the assumptions of part (ii) and Theorem 5.6(ii) we conclude that $F_{V}=\alpha F_{X}+$ $(1-\alpha) F_{Y} \preccurlyeq_{L Z} F_{W}$, hence with (i), $U \leqslant_{L Z} V$, it follows that $U \leqslant_{L Z} W$.

Part (iii) is a consequence of Corollary 5.2

\subsection{Monotone approximation of distributions}

The following theorem shows that, in the lift zonoid order, every probability distribution can be approximated from below by empirical distributions and from above by absolutely continuous distributions.

Theorem 5.8. Given $F \in \mathscr{F}_{0}$, there exists a sequence $\left\{F_{n}\right\}$ of empirical distributions and a sequence $\left\{F^{n}\right\}$ of absolutely continuous distributions such that both converge weakly to $F$ and

$$
F_{n} \preccurlyeq_{L Z} F \preccurlyeq_{L Z} F^{n}
$$

Proof. We show that there exist such sequences with $F_{n} \preccurlyeq_{\text {dil }} F \preccurlyeq_{\text {dil }} F^{n}$; then the theorem follows from Corollary 5.1.

Let $F \in \mathscr{F}_{0} . F$ is decomposed, $F=D+C$, into a discrete measure $D \in \mathscr{C}_{0}$ and an atomless measure $C \in \mathscr{C}_{0}$. First, we approximate $C$, in weak convergence from below, by a sequence $\left\{C_{n}\right\}$ of discrete measures such that $C$ is a dilation of $C_{n}$ for each $n$. We do this as follows. Let $S_{n}=\left\{x: \sum_{j=1}^{d} x_{i} \leqslant n\right\}, n \in \mathbb{N}$, and $\mathscr{S}_{n m}$ be a partition of the simplex $S_{n}$ into simplices of diameter less than $m^{-1}, m \in \mathbb{N}$. Define the measure

$$
C_{n m}(\{y\})= \begin{cases}\int S \mathrm{~d} C(x) & \text { if } y=\int S_{S} x \mathrm{~d} C(x), S \in \mathscr{S}_{n m}, \\ \int_{0} \mathbb{R}^{d} \backslash S_{n} \mathrm{~d} C(x) & \text { if } y=\int_{\mathbb{R}^{d} \backslash S_{n}} x \mathrm{~d} C(x), \\ \text { otherwise. }\end{cases}
$$


Then $C_{n m}$ is discrete and $C_{n m} \preccurlyeq_{\text {dil }} C$ for all $n$ and $m$. Further, $C_{n n} \stackrel{\text { weak }}{\rightarrow} C$. We conclude that $D_{n}:=D+C_{n n} \stackrel{\text { weak }}{\longrightarrow} F$ and $D_{n} \leqslant$ dil $F$ for all $n$.

Now, a discrete distribution $G$ with support $y_{1}, \ldots, y_{m}$ and probabilities $p_{1}, \ldots, p_{m}$ can be approximated by a sequence of empirical distributions $E_{r}$ such that $G$ is a dilation of $E_{r}$ for every $r$. For this we employ empirical distributions whose support includes the support of $G$ and, in addition, a vector $z$. For every $r$, the probability of $y_{i}$ under $E_{r}$ is chosen to be $k_{r i} / r, \quad k_{r i} \in\{0,1, \ldots, r\}$, such that $k_{r i} / r \leqslant p_{i}, \quad\left(k_{r i}+1\right) / r>p_{i}$, and the remaining probability at $z$ equal to $\sum\left(r p_{i}-k_{r i}\right) y_{i} / \sum\left(p_{i} r-k_{r i}\right)$. Then, obviously, $G$ is a dilation of $E_{r}$. This proves the existence of a sequence $\left\{E_{n r}\right\}$ of empirical distributions such that $E_{n r} \stackrel{\text { weak }}{\rightarrow} D_{n}$ and $E_{n r} \preccurlyeq_{\text {dil }} D_{n}$ for all $n$ and $r$, and therefore, by the above, $F_{n}:=E_{n n} \stackrel{\text { weak }}{\rightarrow} F$ and $F_{n} \preccurlyeq_{\text {dil }} F$ for all $n$.

With respect to the approximation from above, we proceed as follows. For $x \in \mathbb{R}^{d}$, and $n \in \mathbb{N}$, let $T_{x}^{n}$ be the multivariate normal distribution with expectation $x$ and covariance matrix $n^{-1} I_{d}$, where $I_{d}$ is the $d \times d$ unit matrix. Then, for every $x, T_{x}^{n}$ is a dilation of the probability measure concentrated at $x$. The probability measure $F^{n}, F^{n}(B)=$ $\int_{\mathbb{R}^{d}} T_{x}^{n}(B) \mathrm{d} F(x)$, as a mixture, is a dilation of $F$; see Phelps (1966, ch. 13). Further, $F^{h}$ is absolutely continuous. Because $F^{h} \stackrel{\text { weak }}{\longrightarrow} F$, the proof is complete.

In the case $d=1$, a given nontrivial $F \in \mathscr{F}_{0}$ can also be approximated the other way round, viz. by a sequence of absolutely continuous distributions from below and a sequence of discrete distributions from above. This holds because any convex centrally symmetric set in $\mathbb{R}^{2}$ is a zonoid, which in higher dimensions is not true. The problem whether $F$ can be approximated in the lift zonoid order from above by empirical distributions is related to the problem of approximating a zonoid by zonotopes on the basis of finitely many support values. This problem is non-trivial and, to our knowledge, unsolved; see Goodey and Weil (1993, p. 1321).

\subsection{An application to random determinants}

Like the dilation order, the lift zonoid order may be used to generate many probability inequalities. Here we present an application to random determinants. Let $X$ be a random vector in $\mathbb{R}^{d}$. Then, by (4.3), the volume of the lift zonoid of $X$ equals $(1 /(d+1) !) \mathrm{E}\left|\operatorname{det} M_{\hat{X}}\right|$, where $M_{\hat{X}}$ is a $(d+1) \times(d+1)$ matrix whose columns are independent and identically distributed copies of $(1, X)$. From the definition of the lift zonoid order we know that $X \leqslant_{L Z} Y$ implies $\hat{Z}(X) \subset \hat{Z}(Y)$, hence

$$
X \preccurlyeq_{L Z} Y \text { implies } \mathrm{E}\left|\operatorname{det} M_{\hat{X}}\right| \leqslant \mathrm{E}\left|\operatorname{det} M_{\hat{Y}}\right| \text {. }
$$

The zonoids of $X$ and $Y$ are projections of the corresponding lift zonoids on their last $d$ coordinates. Therefore the same inequality follows from the zonoid volumes

$$
X \preccurlyeq_{L Z} Y \text { implies } \mathrm{E}\left|\operatorname{det} M_{X}\right| \leqslant \mathrm{E}\left|\operatorname{det} M_{Y}\right|
$$


By this the comparison results of Vitale (1991a; 1991b) can be strengthened. For example, let $Y$ and $Y^{\prime}$ be random vectors with $\mathrm{E}\left[Y^{\prime} \mid Y\right]=0$. Then

$$
\mathrm{E}\left|\operatorname{det} M_{Y}\right| \leqslant \mathrm{E}\left|\operatorname{det} M_{Y+Y^{\prime}}\right| \text {. }
$$

This follows from our Theorem 5.2(vi) and (5.12). It extends Theorem 5.2 in Vitale (1991b). Further, Vitale's Theorem 5.5 is an immediate consequence of our Theorem 5.7(ii).

\section{Acknowledgements}

This research has been supported by a grant from the Deutsche Forschungsgemeinschaft (DFG). We thank two referees and Alfred Müller for their careful reading and useful comments.

\section{References}

Bolker, E.D. (1969) A class of convex bodies. Trans. Amer. Math. Soc., 145, 323-346.

Bonnesen, J. and Fenchel, W. (1934) Theorie der konvexen Körper. Berlin: Springer Verlag.

Efron, B. (1965) The convex hull of a random set of points. Biometrika, 52, 331-343.

Eggleston, H.G. (1958) Convexity. New York: Cambridge University Press.

Goodey, P. and Weil, W. (1993) Zonoids and generalizations. In P.M. Gruber and J.M. Wills (eds), Handbook of Convex Geometry, pp. 1297-1326. Amsterdam: North-Holland.

Helgason, S. (1980) The Radon Transform, Prog. Math. 5. Boston: Birkhäuser.

Koshevoy, G. (1995) Multivariate Lorenz majorization. Soc. Choice and Welf., 12, 93-102.

Laha, R.G. and Rohatgi, V.K. (1979) Probability Theory. New York: Wiley.

Lindenstrauss, J. (1966) A short proof of Liapounoff's convexity theorem. J. Math. Mech. 15, 971972.

Mardia, K.V., Kent, J.T. and Bibby, J.M. (1979) Multivariate Analysis. London: Academic Press.

Mosler, K. and Scarsini, M. (1991) Some theory of stochastic dominance. In K. Mosler and M. Scarsini (eds), Stochastic Orders and Decision under Risk, pp. 261-284. Hayward, CA: Institute of Mathematical Statistics.

Phelps, R.R. (1966) Lectures on Choquet's Theorem. Princeton, NJ: Van Nostrand.

Rényi, A. and Sulanke, R. (1963) Über die konvexe Hülle von $n$ zufällig gewählten Punkten. $Z$. Wahrscheinlichkeitstheorie Verw. Geb., 2, 75-84.

Rényi, A. and Sulanke, R. (1964) Über die konvexe Hülle von $n$ zufällig gewählten Punkten II. $Z$. Wahrscheinlichkeitstheorie Verw. Geb., 3, 138-147.

Rickert, N.W. (1967) The range of a measure. Bull. Amer. Math. Soc., 73, 560-563.

Schneider, R. (1967) Zu einem Problem von Shephard über die Projektionen konvexer Körper. Math. Z., 101, 71-82.

Schneider, R. and Weil, W. (1983) Zonoids and related topics. In P.M. Gruber and J.M. Wills (eds), Convexity and Its Applications, pp. 296-317. Basel: Birkhäuser.

Shaked, M. and Shanthikumar, J.G. (1994) Stochastic Orders and Their Applications. Boston: Academic Press.

Shephard, G.C. (1974) Combinatorial properties of associated zonotopes. Canad. J. Math., 26, 302321. 
Torgersen, E. (1991) Comparison of Statistical Experiments. Cambridge: Cambridge University Press. Vitale, R.A. (1991a) Expectation inequalities from convex geometry. In K. Mosler and M. Scarsini (eds), Stochastic Orders and Decision under Risk, pp. 372-379. Hayward, CA: Institute of Mathematical Statistics.

Vitale, R.A. (1991b) Expected absolute random determinants and zonoids. Ann. Appl. Probab. 1, 293300.

Weil, W. and Wieacker, J.A. (1993) Stochastic geometry. In P.M. Gruber and J.M. Wills (eds) Handbook of Convex Geometry, pp. 1391-1438. Amsterdam: North-Holland.

Received May 1996 and revised December 1996. 